\title{
Factors Associated with Fluid Sequestration in Patients with Acute Pancreatitis: A Prospective Study in Tertiary Centre Hospital in Nepal
}

\author{
Raju Bhandari $\mathbb{D},{ }^{1}$ Krishna Sapkota, ${ }^{2}$ Seema Subedi $\mathbb{D},{ }^{3}$ Som Kumar Shrestha $\mathbb{D},{ }^{4}$ \\ Edward Sutanto $\mathbb{D}^{5}$, Prabhat Jha $\mathbb{D}^{1}{ }^{1}$ and Ramesh Singh Bhandari ${ }^{1}$ \\ ${ }^{1}$ Department of General and Gastrointestinal Surgery, Tribhuvan University Teaching Hospital, Institute of Medicine, \\ Tribhuvan University, Kathmandu, Nepal \\ ${ }^{2}$ Public Health Officer, Health Coordination Division, Ministry of Health and Population, Government of Nepal, Nepal \\ ${ }^{3}$ Global Disease Epidemiology and Control, Department of International Health, Johns Hopkins Bloomberg School of Public Health, \\ Baltimore, USA \\ ${ }^{4}$ Department of Medical Statistics, London School of Hygiene and Tropical Medicine, London, UK \\ ${ }^{5}$ Department of Health Behavior, Roswell Park Comprehensive Cancer Center, Buffalo, USA
}

Correspondence should be addressed to Raju Bhandari; rbiom27@gmail.com

Received 16 January 2021; Revised 3 June 2021; Accepted 13 June 2021; Published 25 June 2021

Academic Editor: Paraskevas Gkolfakis

Copyright (C) 2021 Raju Bhandari et al. This is an open access article distributed under the Creative Commons Attribution License, which permits unrestricted use, distribution, and reproduction in any medium, provided the original work is properly cited.

Background. Acute pancreatitis (AP) is associated with extensive fluid sequestration. The aim of this study was to determine association of fluid sequestration at 48 hours after hospital admission ( $\mathrm{FS}^{48}$ ) in AP patients with demographics, clinical parameters, and outcomes of AP. Methods. A prospective observational study was carried out on all adult patients with AP admitted to Tribhuvan University Teaching Hospital, Nepal, from January to September 2017. FS ${ }^{48}$ was calculated as the difference between fluid input and output in the first 48 hours of admission. The Kruskal-Wallis test with post hoc Dunn's test examined the difference in $\mathrm{FS}^{48}$ between mild AP, moderately severe AP, and severe AP. Linear regression analysis was used to evaluate association between $\mathrm{FS}^{48}$ with patients' characteristics and outcomes of AP. Outcomes of AP assessed included pancreatic necrosis, persistent organ failure, length of stay, and in-hospital mortality. Results. Eighty patients (median age 44 years; $57 \%$ male) with a median $\mathrm{FS}^{48}$ of $1610 \mathrm{~mL}$ were evaluated. The median $\mathrm{FS}^{48}$ for mild AP, moderately severe AP, and severe AP were $1,180 \mathrm{~mL}, 2,380 \mathrm{~mL}$, and $3,500 \mathrm{~mL}$, respectively. There was a significant difference in pairwise comparisons between mild AP and moderately severe AP, along with mild AP and severe AP. Younger age, other etiology, and higher creatinine were independently associated with increased $\mathrm{FS}^{48}$. Increased $\mathrm{FS}^{48}$ was significantly associated with pancreatic necrosis, persistent organ failure, and in-hospital mortality. Conclusions. In our study population, younger age and higher creatinine were predictors of increased $\mathrm{FS}^{48}$. Increased $\mathrm{FS}^{48}$ was associated with poorer outcomes of AP.

\section{Introduction}

Acute pancreatitis (AP), a common surgical presentation, is the most common pancreatic disease with global estimates of incidence and mortality being 33.7 cases per 100,000 personyears and 1.6 deaths per 100,000 person years, respectively [1]. Gallstones and alcohol are the two most common etiological factors for AP, accounting approximately $80 \%$ of all causes, with gallstone pancreatitis being twice as frequent as alcohol pancreatitis [2-4].

Based on the revised Atlanta classification, AP is categorized into mild, moderately severe, and severe [5]. Overall, mortality increased with disease severity, ranging from less than $5 \%$ in mild AP up to $17.8 \%-41.9 \%$ in severe AP $[6,7]$. Supportive care, which includes fluid therapy, bowel rest, and analgesics, is the mainstay of treatment in AP $[8,9]$. As 
fluid sequestration (FS) is commonly seen in patients with AP and associated with worse outcome [10, 11], identification of predictors of FS may help clinician to optimize fluid resuscitation among patients with AP that requires early and aggressive fluid therapy.

Two studies (de-Madaria et al. and Sinha et al.) have examined early predictors of FS in AP $[10,11]$. In both studies, younger age, alcoholic etiology, hemoconcentration, and systemic inflammatory response syndrome (SIRS) were found to be independent predictors of increased fluid sequestration at 48 hours after hospital admission $\left(\mathrm{FS}^{48}\right)[10,11]$. Additionally, hyperglycemia was found to be a significant predictor of $\mathrm{FS}^{48}$ in one study [10]. These retrospective studies however were conducted in developed countries $[10,11]$, yet AP in the developing countries may have a markedly different disease profile to that in developed countries $[12,13]$. While alcohol consumption is a leading cause of AP in developed countries, other causes of AP (such as hypertriglyceridemia or mumps) are more prominent in developing countries $[12,13]$, thus requiring different management to treat underlying causes of AP. Thus, we primarily aimed to validate the association between characteristics of AP patients at admission and $\mathrm{FS}^{48}$ in a developing country setting. Secondarily, we also examined the association between $\mathrm{FS}^{48}$ and outcomes of AP among these patients, along with the difference of $\mathrm{FS}^{48}$ between mild, moderately severe, and severe AP. We used the STROBE statement to guide the writing of this study [14].

\section{Materials and Methods}

2.1. Patient Population. We conducted a prospective cohort study of adult patients (age $\geq 18$ years) with a diagnosis of AP admitted in the surgical wards of Tribhuvan University Teaching Hospital, Nepal, from January to September 2017. In accordance with the revised Atlanta classification, AP was defined as 2 of the following 3 criteria: (1) characteristic abdominal pain, (2) serum amylase and/or lipase greater than 3 times the upper limit of normal, and (3) abdominal imaging findings consistent with AP [5]. We excluded patients undergoing hemodialysis, patients with congestive heart failure, and transferred patients with incomplete record on $\mathrm{FS}^{48}$.

2.2. Variables. The main dependent variable in this study is $\mathrm{FS}^{48}$. Similar with previous studies $[10,11]$, we calculated $\mathrm{FS}^{48}$ as the difference between the total fluid input and total fluid output in the first 48 hours of admission. Fluid input included administration of all intravenous crystalloid or colloid preparations, blood products, antibiotics, and oral fluid intake. Fluid output included recorded volumes of vomitus, urine, stool, and insensible losses ( $10 \mathrm{mg}$ per $\mathrm{kg}$ body weight per day). Additionally, $500 \mathrm{~mL}$ was added to the fluid output if patients had a temperature $>37.8^{\circ} \mathrm{C}$ per day.

Demographic characteristics (age and sex) and clinical parameters (etiology, SIRS at presentation, hematocrit, total leukocyte count, serum glucose, serum creatinine, blood urea nitrogen (BUN), and serum sodium) were collected. Etiology was categorized into alcohol, gallstones, and other.
As it is possible for patient to have more than one etiology of AP, etiology was treated as a nonmutually exclusive categorical variable. SIRS is defined as the occurrence by two or more of the following conditions: (1) temperature $>38^{\circ} \mathrm{C}$ or $<36^{\circ} \mathrm{C}$, (2) heart rate $>90$ beats per minute, (3) respiratory rate $>20$ breaths per minute, and (4) white blood cell count $>12,000 / \mathrm{mm}^{3},<4,000 / \mathrm{mm}^{3}$, or $>10 \%$ immature (band) forms [15].

We also collected data on complications of AP (persistent organ failure and pancreatic necrosis), in-hospital mortality, and length of stay. Persistent organ failure (POF) was defined as a Marshall score of 2 or greater in the renal, pulmonary, and or cardiovascular system for longer than 48 hours [5]. Pancreatic necrosis was defined according to the revised Atlanta classification [5] and evaluated using abdominal contrast-enhanced computed tomography (CECT). Abdominal CECT was indicated in the following situations in this study: (1) if there is diagnostic uncertainty; (2) to distinguish interstitial from necrotizing pancreatitis in patients with severe AP; (3) in patients with organ failure, a sign of sepsis or progressive clinical deterioration; and (4) when a localized complication (such as fluid collection, pseudocyst, or pseudoaneurysm) is suspected [16]. We classified patients based on severity of AP into mild AP (MAP), moderately severe AP (MSAP), and severe AP (SAP) in accordance to the revised Atlanta classification [5].

2.3. Study Endpoint. The primary study endpoint is $\mathrm{FS}^{48}$ outcome, while the secondary study endpoints are POF, pancreatic necrosis, in-hospital mortality, and length of stay outcomes.

2.4. Statistical Analysis. Demographic characteristics, clinical parameters, and outcomes of AP were reported using descriptive statistics. Three separate analyses were done in accordance to our aims. First, as $\mathrm{FS}^{48}$ did not follow normal distribution, the Kruskal-Wallis test was employed to examine differences in $\mathrm{FS}^{48}$ between MAP, MSAP, and SAP. Multiple pairwise comparisons were addressed by using Dunn's test. Second, simple and multiple linear regressions were used to evaluate the association between potential predictors at admission and $\mathrm{FS}^{48}$. Third, simple and multiple linear regressions were used to evaluate the association between $\mathrm{FS}^{48}$ and outcomes of AP. In both regression analyses, we reported $\beta$ coefficient, which represented change $(\mathrm{mL})$ in $\mathrm{FS}^{48}$ associated with unit change in predictor variables, and 95\% confidence interval. All statistical analyses were performed in Stata version 15.0 (StataCorp, College Station, Texas, United States). All tests were two-tailed and considered significant at $p<0.05$.

\section{Results}

A total of 80 patients were recruited in this study. Table 1 describes baseline characteristics and outcomes in our study cohort. The median age was 44 years, and majority were male (57.5\%). Gallstone was the most common etiology $(80.0 \%)$. Alcohol etiology was present in approximately a quarter of study cohort (22.5\%), while other etiology (3 cases) included 
TABle 1: Demographics characteristics, clinical parameters, and outcomes of the study cohort.

\begin{tabular}{|c|c|}
\hline & $N=80$ \\
\hline \multicolumn{2}{|l|}{ Demographics } \\
\hline Age (years) & $44(36-56.5)$ \\
\hline Male & $46(57.5 \%)$ \\
\hline \multicolumn{2}{|l|}{ Clinical characteristics } \\
\hline $\mathrm{FS}^{48}(\mathrm{~mL})$ & $1610(810-3575)$ \\
\hline \multicolumn{2}{|l|}{ Etiology* } \\
\hline Alcohol & $18(22.5 \%)$ \\
\hline Gallstones & $64(80.0 \%)$ \\
\hline Others & $3(3.7 \%)$ \\
\hline SIRS at presentation & $34(42.5 \%)$ \\
\hline Hematocrit (\%) & $40.0(35.2-44.5)$ \\
\hline Total leukocyte count $\left(/ \mathrm{mm}^{3}\right)$ & $11350(8000-14750)$ \\
\hline Glucose (mg/dL) & $143.1(111.6-173.7)$ \\
\hline Creatinine (mg/dL) & $0.91(0.78-1.33)$ \\
\hline BUN (mmol/L) & $5.0(3.5-9.1)$ \\
\hline Sodium (mmol/L) & $138.3(135.0-141.5)$ \\
\hline \multicolumn{2}{|l|}{ Outcomes } \\
\hline \multicolumn{2}{|l|}{ Pancreatic necrosis $(n=23)$} \\
\hline None & $12(52.2 \%)$ \\
\hline$\leq 30$ & $7(30.4 \%)$ \\
\hline$>30$ & $4(17.4 \%)$ \\
\hline Persistent organ failure & $15(18.8 \%)$ \\
\hline In-hospital mortality & $2(2.5 \%)$ \\
\hline Length of stay (days) & $4.5(3.0-8.5)$ \\
\hline \multicolumn{2}{|l|}{ Severity of acute pancreatitis } \\
\hline Mild & $54(67.5 \%)$ \\
\hline Moderately severe & $11(13.7 \%)$ \\
\hline Severe & $15(18.8 \%)$ \\
\hline
\end{tabular}

Values are expressed in median (interquartile range) or $n(\%) .{ }^{*}$ Add up to more than $100 \%$ as more than one etiology may be identified in a patient. Abbreviations: BUN: blood urea nitrogen; $\mathrm{FS}^{48}$ : fluid sequestration at 48 hours after hospital admission; SIRS: systemic inflammatory response syndrome.

one each of idiopathic, pancreatic malignancy, and hypertriglyceridemia. Nearly one-fifth developed persistent organ failure and $2.5 \%$ died during hospitalization. Abdominal contrast-enhanced computed tomography was performed in 23 patients to evaluate pancreatic necrosis. Among these 23 patients, almost half have a degree of pancreatic necrosis (47.8\%). The median value of $\mathrm{FS}^{48}$ was $1610 \mathrm{~mL}$ (interquartile range (IQR): $810-3575 \mathrm{~mL}$ ).

Figure 1 shows the difference in $\mathrm{FS}^{48}$ between MAP, MSAP, and SAP. The median $\mathrm{FS}^{48}$ for MAP, MSAP, and SAP were $1180 \mathrm{~mL}$ (IQR: $730-2240 \mathrm{~mL}$ ), $2380 \mathrm{~mL}$ (IQR: 950-7280 mL), and $3500 \mathrm{~mL}$ (IQR: $1920-8110 \mathrm{~mL}$ ), respectively. The Kruskal-Wallis test showed there was a significant difference in $\mathrm{FS}^{48}$ between these three groups $(p<0.01)$. Post hoc analysis showed that there was significant difference for pairwise comparisons of MAP versus MSAP $(p<0.05)$ and MAP versus SAP $(p<0.01)$.

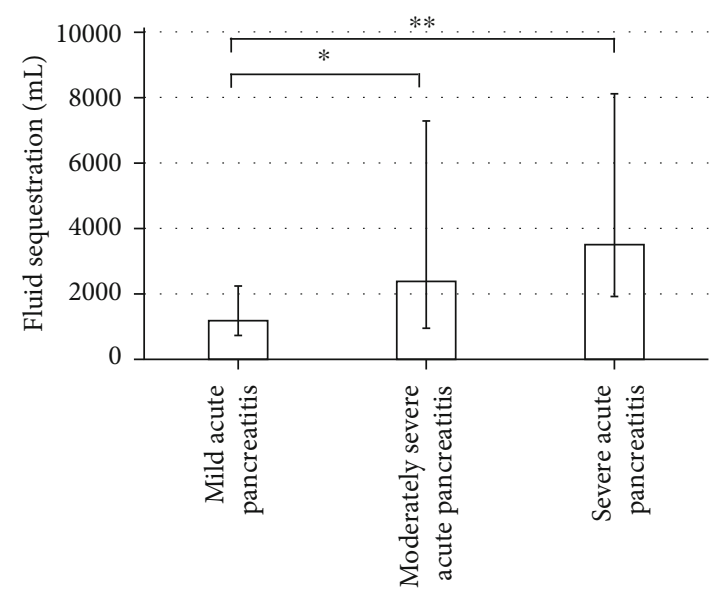

FIgURE 1: Fluid sequestration in the first 48 hours from admission between mild, moderately severe, and severe acute pancreatitis. The values represent median and error bars are interquartile range. ${ }^{*} p<0.05$ and ${ }^{* *} p<0.01$ (Dunn's test).

Table 2 displays the association between patient characteristics and $\mathrm{FS}^{48}$. Younger age, other etiology, and higher creatinine were independently associated with increased $\mathrm{FS}^{48}$. Table 3 displays the association between $\mathrm{FS}^{48}$ and outcomes of AP. Although length of stay was not associated with $\mathrm{FS}^{48}, \mathrm{FS}^{48}$ was associated with pancreatic necrosis, persistent organ failure, and in-hospital mortality.

\section{Discussions}

This prospective study evaluated predictors and outcomes associated with increased $\mathrm{FS}^{48}$ among 80 patients with AP in a developing country setting. There are three key findings in this study. First, there is a significant increase in median $\mathrm{FS}^{48}$ in MSAP and SAP compared to MAP. Second, we found that younger age, other etiology, and higher creatinine predict increased $\mathrm{FS}^{48}$. Third, increased $\mathrm{FS}^{48}$ is associated with worse patient outcomes.

Previous study by Ranson et al. in 1974 reported a mean $\mathrm{FS}^{48}$ of 3.7 L and 5.6 L for MAP and SAP, respectively [17]. At the time of publication of the study, there was no classification of MSAP for severity of AP. A study by de-Madaria et al. reported the median $\mathrm{FS}^{48}$ was $3.0 \mathrm{~L}$ (IQR: $1.5-5.0 \mathrm{~L}$ ) and $6.4 \mathrm{~L}$ (IQR: 3.6-9.5 L) in those without necrosis and those with necrosis [10], while a study by Sinha et al. reported median FS was 4.0 L (IQR: 2.0-5.9L) and 9.2 L (IQR: 4.813.2 L) in those without POF and those with POF [11]. While both studies did not report median $\mathrm{FS}^{48}$ based on severity of AP specifically, both necrosis and POF are criteria for MSAP and SAP [5]. Thus, our finding that there is a significant increase in median $\mathrm{FS}^{48}$ in MSAP and SAP compared to MAP is consistent with previous studies.

Although studies on AP have been conducted in Nepal, there is no study that has examined FS among patients with AP. A previous study from our center noted biochemical markers, total serum calcium and albumin-corrected calcium, are useful severity predictors in AP [18]. Our study further adds that median $\mathrm{FS}^{48}$ is a useful parameter as increased 
TABLE 2: Association between demographic characteristics and clinical parameters with fluid sequestration in the first 48 hours from admission.

\begin{tabular}{|c|c|c|}
\hline Variables & Unadjusted $\beta$ coefficient (95\% CI) & Adjusted $\beta$ coefficient (95\% CI) \\
\hline \multicolumn{3}{|l|}{ Demographics } \\
\hline Age (years) & $-20.8(-58.8,17.1)$ & $-45.2(-72.1,-18.4)^{* *}$ \\
\hline Female & $-196.6(-1392.3,999.0)$ & $-1.5(-952.9,949.9)$ \\
\hline \multicolumn{3}{|l|}{ Clinical characteristics } \\
\hline Alcohol etiology & $-1520.6(-2894.9,-146.3)^{*}$ & $-754.5(-1954.4,445.4)$ \\
\hline Gallstones etiology & $1379.1(-66.6,-2824.7)$ & $589.5(-609.1,1788.1)$ \\
\hline Other etiology & $3411.1(394.3,6427.9)^{*}$ & $4591.4(2474.0,6708.8)^{* * *}$ \\
\hline SIRS at presentation & $1037.1(-136.3,2210.5)$ & $21.4(-824.9,867.7)$ \\
\hline Hematocrit (\%) & $100.9(8.3,193.5)^{*}$ & $51.9(-17.8,121.7)$ \\
\hline Glucose (mg/dL) & $10.0(0.5,19.5)^{*}$ & $2.9(-4.4,10.2)$ \\
\hline Creatinine (mg/dL) & $3124.6(2333.2,3916.1)^{* * *}$ & $2943.8(2061.7,3825.9)^{* * *}$ \\
\hline $\mathrm{BUN}(\mathrm{mmol} / \mathrm{L})$ & $174.0(58.8,289.3)^{* *}$ & $-3.7(-101.2,93.7)$ \\
\hline Sodium (mmol/L) & $97.8(-20.4,215.9)$ & $43.5(-38.6,125.7)$ \\
\hline
\end{tabular}

TABLE 3: Association between outcomes of AP and FS in the first 48 hours from admission.

\begin{tabular}{lcr}
\hline Variables & Unadjusted $\beta$ coefficient $(95 \%$ CI $)$ & Adjusted $\beta$ coefficient $(95 \%$ CI $)$ \\
\hline $\begin{array}{l}\text { Pancreatic necrosis } \\
\text { None }\end{array}$ & Ref & Ref \\
$\leq 30$ & $2270.4(261.7,4279.0)^{*}$ & $4182.0(1373.0,6990.9)^{* *}$ \\
$>30$ & $4035.0(1596.6,6473.4)^{* *}$ & $6088.7(3683.4,8494.1)^{* * *}$ \\
Persistent organ failure & Ref & Ref \\
$\quad$ No & $1893.6(439.6,3347.6)^{*}$ & $-3192.1(-5571.2,-813.0)^{*}$ \\
Yes & & \\
In-hospital mortality & Ref & Ref \\
$\quad$ No & $6076.7(2544.7,9608.9)^{* *}$ & $5956.5(2523.1,9389.9)^{* *}$ \\
Yes & $101.4(19.7,183.0)^{*}$ & $19.8(-64.8,104.8)$ \\
Length of stay & &
\end{tabular}

median $\mathrm{FS}^{48}$ was observed in more severe AP. SAP has been noted to be associated with vascular leak syndrome [19], which increased systemic vascular permeability leading to extravasation of fluids and proteins into tissues, thus increasing FS.

Consistent with previous studies $[10,11]$, our study confirms that younger age is associated with increased $\mathrm{FS}^{48}$. It is hypothesized that, due to concern of volume overload, older patients may receive less aggressive fluid resuscitation resulting in lower FS [10]. While previous studies found alcohol etiology to be predictors of increased $\mathrm{FS}^{48}[10,11]$, we found other etiologies of AP to be significantly associated with increased $\mathrm{FS}^{48}$. Additionally, we did not find hematocrit, glucose, and presence of SIRS to be predictors of increased FS ${ }^{48}$ in our study cohort. These discrepancies may be explained partly due to differences between our study cohort and patients from previous studies. Unlike our study where majority of AP etiology was gallstones, both previous studies have a markedly lower gallstones etiology (de-Madaria et al.: $41.4 \%$ and Sinha et al.: $13.2 \%)[10,11]$. Secondly, our study was conducted in low-resource setting which influenced on medical imaging modalities performed to our study cohort. Lastly, our relatively small sample size may not allow us to have enough power to detect difference in increased $\mathrm{FS}^{48}$.

Higher creatinine was found to be independent predictors of increased $\mathrm{FS}^{48}$ in our study cohort. Both creatinine and BUN are well-known markers of renal function. Creatinine, however, have been hypothesized to be less sensitive to small changes in intravascular volume and better reflect visceral organ injury [20]. As kidney injury is a common complication of AP, with prevalence around $20 \%$ in all AP patients and up to $70 \%$ in SAP, it can be resulted from volume depletion due to fluid sequestration [21, 22]. Multiple studies also have demonstrated that elevated serum creatinine level is associated with the development of pancreatic necrosis, POF, and mortality [20, 23, 24]. 
We found $\mathrm{FS}^{48}$ to be significantly associated with several outcomes of AP, including POF, pancreatic necrosis, and inhospital mortality, but not with increased length of stay. While $\mathrm{FS}^{48}$ was significantly associated with POF consistently across studies $[10,11]$, there is a mixed result for pancreatic necrosis and length of stay. It is important to note that only the presence of POF is used to define severe AP, and studies have reported that POF was the strongest predictor of mortality in necrotizing pancreatitis $[5,25]$. While deMadaria's study reported increased $\mathrm{FS}^{48}$ was associated with pancreatic necrosis and length of stay, Sinha et al. did not find these associations $[10,11]$. This mixed result may be attributed to the difference rates of these complications occurring in each study cohort. Our study also reinforces finding from a previous study that fluid sequestration of $2 \mathrm{~L}$ or more per day, and lasting longer than 48 hour, is an accurate and simple predictor of mortality in AP [26].

To the best of our knowledge, this is the first study from a developing country that examined association between increased $\mathrm{FS}^{48}$, patients' characteristics, and outcomes of AP. While the prospective nature of this study is a strength, there are several limitations in this study. First, the sample size is relatively small. Although we included all eligible patients for 9 months, we were only able to recruit 80 patients to our study cohort. Second, the study was conducted in a single tertiary hospital in Nepal. As our center is a major referral hospital in Nepal, more severe AP patients were recruited in this study; thus, it may limit the generalizability of our findings. Lastly, similar with Sinha et al.'s study [11], we also did not perform abdominal CECT in all patients; thus, the rates of pancreatic necrosis or acute fluid collection may be underreported. However, abdominal CECT is not routinely indicated in AP management as unnecessary imaging studies, especially in the early hospital course, is associated with increased radiation dose for patients and high health care costs, frequently without impact on patient outcomes or management [16, 27-29]. Nevertheless, our study provides an important external validation of the results from de-Madaria et al.'s and Sinha et al.'s studies in the context of low-resource settings.

\section{Conclusions}

In conclusion, younger age and higher creatinine were predictors of increased $\mathrm{FS}^{48}$ in our study cohort. As increased $\mathrm{FS}^{48}$ was associated with poorer outcomes of AP, there is a need to develop a simple scoring system that incorporates easily obtained variables at presentation to reliably predict FS for AP patients.

\section{Abbreviations}

AP: Acute pancreatitis

BUN: Blood urea nitrogen

CECT: Contrast-enhanced computed tomography

CI: Confidence interval

$\mathrm{FS}^{48}$ : Fluid sequestration in the first 48 hours from admission

IQR: Interquartile range
MAP: Mild acute pancreatitis

MSAP: Moderately severe acute pancreatitis

SAP: Severe acute pancreatitis

SIRS: Systemic inflammatory response syndrome.

\section{Data Availability}

The dataset used for the current study is available from the corresponding author on reasonable request.

\section{Ethical Approval}

This study was approved by Institutional Review Board of Institute of Medicine Tribhuvan University.

\section{Consent}

All participants included in the study provided written informed consent.

\section{Conflicts of Interest}

The authors declare that they have no relevant conflicts of interests.

\section{Authors' Contributions}

$\mathrm{RB}$ initiated study conception and design. RB performed the data collection. RB, KS, SS, SKS, and ES performed the data analysis. RB prepared the first draft of the manuscript. ES, PJ, and RSB helped prepare the final manuscript. All authors have read and approved the final manuscript.

\section{References}

[1] A. Y. Xiao, M. L. Y. Tan, L. M. Wu et al., "Global incidence and mortality of pancreatic diseases: a systematic review, metaanalysis, and meta-regression of population-based cohort studies," The Lancet Gastroenterology \& Hepatology, vol. 1, no. 1, pp. 45-55, 2016.

[2] M. B. Zilio, T. F. Eyff, A. L. F. Azeredo-Da-Silva, V. P. Bersch, and A. B. Osvaldt, "A systematic review and meta-analysis of the aetiology of acute pancreatitis," vol. 21, no. 3, pp. 259$267,2019$.

[3] G. H. Sakorafas and A. G. Tsiotou, "Etiology and pathogenesis of acute pancreatitis: current concepts," Journal of Clinical Gastroenterology, vol. 30, no. 4, pp. 343-356, 2000.

[4] M. Nesvaderani, G. D. Eslick, D. Vagg, S. Faraj, and M. R. Cox, "Epidemiology, aetiology and outcomes of acute pancreatitis: a retrospective cohort study," International Journal of Surgery, vol. 23, no. Part A, pp. 68-74, 2015.

[5] P. A. Banks, T. L. Bollen, C. Dervenis et al., "Classification of acute pancreatitis -2012: revision of the Atlanta classification and definitions by international consensus," Gut, vol. 62, no. 1, pp. 102-111, 2013.

[6] G. Sarri, Y. Guo, I. Iheanacho, and J. Puelles, "Moderately severe and severe acute pancreatitis: a systematic review of the outcomes in the USA and European Union-5," BMJ Open Gastroenterology, vol. 6, no. 1, article e000248, 2019. 
[7] G. Gravante, G. Garcea, S. L. Ong et al., "Prediction of mortality in acute pancreatitis: a systematic review of the published evidence," Pancreatology, vol. 9, no. 5, pp. 601-614, 2009.

[8] S. Tenner, J. Baillie, J. Dewitt, and S. S. Vege, “American College of Gastroenterology guideline: management of acute pancreatitis," The American Journal of Gastroenterology, vol. 108, no. 9, pp. 1400-1415, 2013.

[9] J. A. Greenberg, J. Hsu, M. Bawazeer et al., "Clinical practice guideline: management of acute pancreatitis," Canadian Journal of Surgery, vol. 59, no. 2, pp. 128-140, 2016.

[10] E. de-Madaria, P. A. Banks, N. Moya-Hoyo et al., "Early factors associated with fluid sequestration and outcomes of patients with acute pancreatitis," Clinical Gastroenterology and Hepatology, vol. 12, no. 6, pp. 997-1002, 2014

[11] A. Sinha, N. Q. Vázquez, M. Faghih et al., "Early predictors of fluid sequestration in acute pancreatitis: a validation study," Pancreas, vol. 45, no. 2, pp. 306-310, 2016.

[12] K. U. Ahmed, M. A. Ahad, M. A. Alim, and A. S. Ekram, "Clinical profile of acute pancreatitis in a teaching hospital," Bangladesh Medical Journal Khulna, vol. 49, no. 1-2, pp. 7-12, 2017.

[13] J. Fan, L. Ding, Y. Lu, J. Zheng, Y. Zeng, and C. Huang, "Epidemiology and etiology of acute pancreatitis in urban and suburban areas in Shanghai: a retrospective study," Gastroenterology Research and Practice, vol. 2018, 8 pages, 2018.

[14] J. P. Vandenbroucke, E. von Elm, D. G. Altman et al., "Strengthening the Reporting of Observational Studies in Epidemiology (STROBE): explanation and elaboration," International Journal of Surgery, vol. 12, no. 12, pp. 1500-1524, 2014.

[15] R. C. Bone, R. A. Balk, F. B. Cerra et al., "Definitions for sepsis and organ failure and guidelines for the use of innovative therapies in sepsis," Chest, vol. 101, no. 6, pp. 1644-1655, 1992.

[16] N. S. Williams, C. J. K. Bulstrod, and P. R. O'Connell, Acute and chronic pancreatitis. Bailey \& Love's Short Practice of Surgery. 26th edition, pp. 1130-1153, CRC Press., 2014.

[17] J. H. Ranson, K. M. Rifkind, D. F. Roses, S. D. Fink, K. Eng, and F. C. Spencer, "Prognostic signs and the role of operative management in acute pancreatitis," Surgery, Gynecology \& Obstetrics, vol. 139, no. 1, pp. 69-81, 1974.

[18] A. Pokharel, P. R. Sigdel, S. Phuyal, P. B. S. Kansakar, and P. Vaidya, "Prediction of severity of acute pancreatitis using total serum calcium and albumin-corrected calcium: a prospective study in tertiary center hospital in Nepal," Surgery Research and Practice, vol. 2017, 5 pages, 2017.

[19] D. C. Whitcomb, V. Muddana, C. J. Langmead et al., “Angiopoietin-2, a regulator of vascular permeability in inflammation, is associated with persistent organ failure in patients with acute pancreatitis from the United States and Germany," The American Journal of Gastroenterology, vol. 105, no. 10, pp. 2287-2292, 2010.

[20] V. Muddana, D. C. Whitcomb, A. Khalid, A. Slivka, and G. I. Papachristou, "Elevated serum creatinine as a marker of pancreatic necrosis in acute pancreatitis," The American Journal of Gastroenterology, vol. 104, no. 1, pp. 164-170, 2009.

[21] J. Wajda, P. Dumnicka, M. Maraj, P. Ceranowicz, M. Kuźniewski, and B. Kuśnierz-Cabala, "Potential prognostic markers of acute kidney injury in the early phase of acute pancreatitis," International Journal of Molecular Sciences, vol. 20, no. 15, pp. 3714-3720, 2019.

[22] M. R. Lattanzio and N. P. Kopyt, “Acute kidney injury: new concepts in definition, diagnosis, pathophysiology, and treat- ment," The Journal of the American Osteopathic Association, vol. 109, no. 1, pp. 13-19, 2009.

[23] M. Lipinski, A. Rydzewski, and G. Rydzewska, "Early changes in serum creatinine level and estimated glomerular filtration rate predict pancreatic necrosis and mortality in acute pancreatitis: creatinine and eGFR in acute pancreatitis," Pancreatology, vol. 13, no. 3, pp. 207-211, 2013.

[24] J. Wan, W. Shu, W. He et al., "Serum creatinine level and APACHE-II score within $24 \mathrm{~h}$ of admission are effective for predicting persistent organ failure in acute pancreatitis," Gastroenterology Research and Practice, vol. 2019, 9 pages, 2019.

[25] Q. Guo, A. Li, Q. Xia et al., "The role of organ failure and infection in necrotizing pancreatitis: a prospective study," Annals of Surgery, vol. 259, no. 6, pp. 1201-1207, 2014.

[26] P. Sauven, M. J. Playforth, M. Evans, and A. V. Pollock, "Fluid sequestration: an early indicator of mortality in acute pancreatitis," The British Journal of Surgery, vol. 73, no. 10, pp. 799800, 1986.

[27] K. J. Mortele, I. K. Ip, B. U. Wu, D. L. Conwell, P. A. Banks, and R. Khorasani, "Acute pancreatitis: imaging utilization practices in an urban teaching hospital-analysis of trends with assessment of independent predictors in correlation with patient outcomes," Radiology, vol. 258, no. 1, pp. 174-181, 2011.

[28] B. W. Spanier, Y. Nio, R. W. van der Hulst, H. A. Tuynman, M. G. Dijkgraaf, and M. J. Bruno, "Practice and yield of early CT scan in acute pancreatitis: a Dutch observational multicenter study," Pancreatology, vol. 10, no. 2-3, pp. 222-228, 2010.

[29] A. P. C. Rocha, K. Schawkat, and K. J. Mortele, "Imaging guidelines for acute pancreatitis: when and when not to image," Abdominal Radiology, vol. 45, no. 5, pp. 1338-1349, 2020 . 